\title{
Objective autorefraction in posterior chamber pseudophakia
}

\author{
Palaniswamy Sunder Raj, Tayo Akingbehin, Anthony M Levy
}

\begin{abstract}
Automated refraction with the Canon RK-1 Autoref keratometer was evaluated in 110 eyes (110 patients) six to eight weeks after they had undergone extracapsular cataract extraction with posterior chamber intraocular lens implantation and achieved a best corrected visual acuity of at least 6/12. Autorefraction readings were obtained in $100(91 \%)$ of these eyes. The agreement between autorefraction and clinical refraction data was $98 \%$ for spherical equivalence $<0.51$ dioptres (D), $95 \%$ for sphere power $<0.51 \mathrm{D}, 94 \%$ for cylinder power $<0.51 D$, and $85 \%$ for cylinder axis $<11^{\circ}$. Autorefraction can provide acceptably accurate postoperative refraction values in pseudophakic eyes.
\end{abstract}

Autorefractors (AR) are being increasingly used in busy ophthalmology clinics because they can be operated by a nurse or technician, ' are much quicker than manual refraction, ${ }^{12}$ and are readily accepted by most patients.' Recent experience indicates that objective autorefraction provides reliable and valid preliminary refraction data, ${ }^{1-12}$ especially in subjects who cannot accommodate owing to aphakia ${ }^{213}$ or cycloplegia. ${ }^{121415}$

Intraocular lenses (IOL) can interfere with the accuracy of objective ARs ${ }^{19^{10}}$ by scattering the measuring infrared beam and increasing 'noise'. ${ }^{16}$ Attempts have been made to overcome this problem in modern ARs by providing an 'IOL setting' which can be switched on to improve the signal-to-noise ratio in the recording system while refracting pseudophakes. We evaluated the use of one such instrument, the Canon RK-1 Autoref keratometer, in patients with posterior chamber IOLs prior to its introduction into routine clinical use.

\section{Patients and methods}

\section{INSTRUMENT}

The Canon RK-1 Autoref keratometer combines both automated refraction and keratometry in one instrument (Fig 1). In the autorefraction mode infrared rays are projected on to the patient's retina and the reflected illumination is detected by an array of photodetectors. This is based on the Scheiner double pinhole principle, which enables the AR to determine the ocular refraction in three meridians. The instrument then computes and displays the objective ocular refraction in terms of sphere, cylinder, and axis corrected for a vertex distance of $12 \mathrm{~mm}$.

The AR covers a pupillary area of $2.9 \mathrm{~mm}$ and can measure up to 15 dioptre (D) sphere, $7 \mathrm{D}$ cylinder, and axis between $1^{\circ}$ and $180^{\circ}$ in incre-

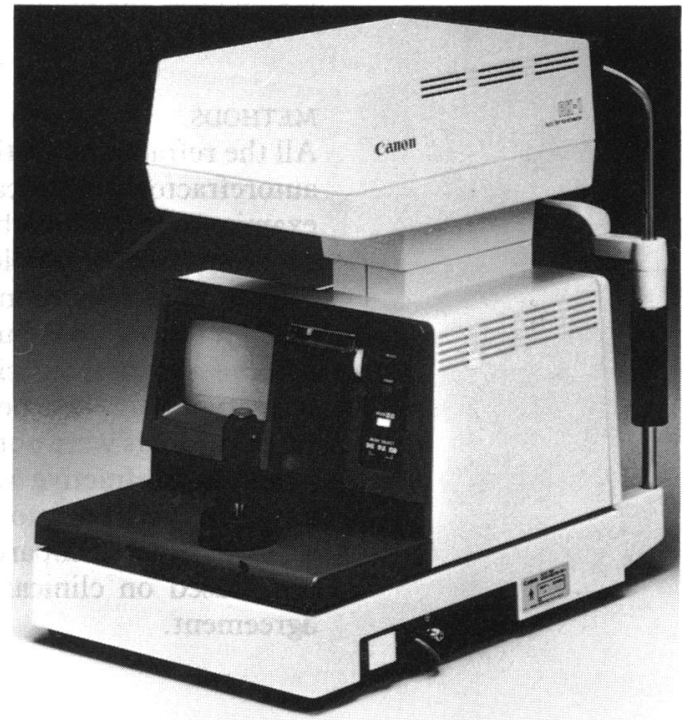

Figure 1 The Canon RK-1 Autoref keratometer

ments of $0.12 \mathrm{D}$ or $1^{\circ}$. It has an in-built autofogging mechanism consisting of a phototarget which is supposed to relax completely the patients' accommodation. An IOL setting is present which can be switched on to improve the accuracy of refraction in pseudophakes.

The measurement of refraction is made in 0.05 second, which is facilitated by sensor assisted alignment and focusing over a built-in television monitor. The iris of the eye to be measured is clearly focused, the ring target is aligned with the pupil, and the measurement button pressed to display the objective ocular refraction. Any number of such measurements may be performed either in the single or continuous mode. The refractive data displayed can be printed out if necessary. If more than three measurements have been performed the computer automatically calculates and prints the most reliable or 'standard' values from the last three to five data.

Low reliability of the measurement due to movement of the eye during testing, postoperative deformation of the cornea or iris, and eccentricity of the IOL are indicated by a special 'reliability mark' (henceforth referred to as 'less reliable' data). Improper alignment, droopy eyelashes or eyelids, and insufficient pupillary size require appropriate remedial action when the signal error is displayed on the television monitor, as valid readings cannot otherwise be obtained. More detailed operating and maintenance information is available in the accompanying instrument manual.

\section{PATIENTS AND EYES}

This was a prospective study carried out between 
six and eight weeks following extracapsular cataract extraction with the implantation of a posterior chamber IOL. When both the eyes of a patient fulfilled the above criteria, only the right eye was studied. Of the 129 such patients seen consecutively in the eye clinic 19 were excluded because their studied eyes did not attain a best corrected visual acuity of at least $6 / 12$. Valid AR values could not be obtained for various reasons in 10 patients, leaving 100 eyes of 100 patients which were analysed.

\section{METHODS}

All the refractions, both clinical as well as on the autorefractor, were carried out by the same examiner (PSR) without the use of either mydriatics or cycloplegics. Objective clinical refraction (streak retinoscopy) was first carried out, followed by subjective verification to achieve the best possible visual acuity. Autorefraction was then performed, and a series of three readings were obtained from each eye. The 'standard' refractive value in the AR printout data (categorised into reliable and less reliable data) was then compared with the final prescription based on clinical refraction for degree of agreement.

\section{STATISTICAL ANALYSIS}

The statistical significance of the difference between the less reliable (indicated by the reliability mark) and reliable autorefractive data

Table 1 Degree of agreement between autorefractive and clinical refractive data of various refractive components

\begin{tabular}{llll}
\hline & \multicolumn{3}{l}{ Autorefractor reading; no(\%) } \\
\cline { 2 - 4 } & $\begin{array}{l}\text { Reliable } \\
(n=70)\end{array}$ & $\begin{array}{l}\text { Less reliable } \\
(n=30)\end{array}$ & $\begin{array}{c}\text { Total } \\
(n=100)\end{array}$ \\
Refractive component & & \\
\hline Spherical equivalent & & & \\
$\pm 0 \cdot 25 \mathrm{D}$ & $57(81 \%)$ & $19(63 \%)$ & 76 \\
$\pm 0 \cdot 50 \mathrm{D}$ & $70(100 \%)$ & $28(93 \%)$ & 98 \\
$\pm 1 \cdot 00 \mathrm{D}$ & $70(100 \%)$ & $30(100 \%)$ & 100 \\
Sphere component & & & \\
$\pm 0 \cdot 25 \mathrm{D}$ & $56(80 \%)$ & $18(60 \%)$ & 74 \\
$\pm 0 \cdot 50 \mathrm{D}$ & $70(100 \%)$ & $25(83 \%)$ & 95 \\
$\pm 1 \cdot 00 \mathrm{D}$ & $70(100 \%)$ & $30(100 \%)$ & 100 \\
Cylinder component & & & \\
$\pm 0 \cdot 25 \mathrm{D}$ & $53(76 \%)$ & $18(60 \%)$ & 71 \\
$\pm 0 \cdot 50 \mathrm{D}$ & $70(100 \%)$ & $24(80 \%)$ & 94 \\
$\pm 1 \cdot 00 \mathrm{D}$ & $70(100 \%)$ & $29(99 \%)$ & 99 \\
Cylinder axis & & & \\
$\pm 5^{\circ}$ & $33(47 \%)$ & $10(33 \%)$ & 43 \\
$\pm 10^{\circ}$ & $63(90 \%)$ & $22(73 \%)$ & 85 \\
$\geqslant 11^{\circ}$ & $3(4 \%)$ & $5(17 \%)$ & 8 \\
$\star$ NC & $4(6 \%)$ & $3(10 \%)$ & 7 \\
\hline
\end{tabular}

$\mathrm{n}=$ Number of eyes (patients)

$\star$ No cylinder in one of the methods of refraction.

Table 2 Mean difference and standard deviations $(S D)$ between autorefraction and clinical refraction

\begin{tabular}{llcl}
\hline & \multicolumn{2}{l}{ Autorefractor reading $;$ mean $(S D)$} \\
\cline { 2 - 4 } Refractive component & Reliable $(n=70)$ & Less reliable $(n=30)$ & Total $(n=100)$ \\
\hline Spherical equivalent (D) & $-0 \cdot 01(0 \cdot 11)$ & $-0 \cdot 02(0 \cdot 37)$ & $-0 \cdot 01(0 \cdot 29)$ \\
Sphere component (D) & $-0 \cdot 03(0 \cdot 23)$ & $-0 \cdot 08(0 \cdot 46)$ & $-0 \cdot 04(0 \cdot 45)$ \\
Cylinder component (D) & $0 \cdot 09(0 \cdot 40)$ & $0 \cdot 15(0 \cdot 45)$ & $0 \cdot 12(0 \cdot 40)$ \\
Cylinder axis (degrees) & $4 \cdot 1(3 \cdot 2)$ & $6 \cdot 1(5 \cdot 4)$ & $4 \cdot 7(6 \cdot 1)$ \\
\hline
\end{tabular}

$\mathrm{n}=$ Number of eyes (patients).

ॠThe minus $(-)$ sign indicates more myopia or less hypermetropia on the autorefractor than by clinical refraction. was evaluated by (1) $\chi^{2}$ test on the number of eyes (patients) in the two groups within $0.51 \mathrm{D}$ of spherical equivalence, spherical power, or cylindrical power and within $11^{\circ}$ in cylindrical axis as determined by clinical refraction; and (2) standard error of the difference between means on the mean difference in the various refractive components between automated and conventional refraction.

\section{Results}

For the 110 eyes (110 patients) with posterior chamber IOLs which were eligible for the study valid autorefractive data could not be obtained in 10 eyes (10 patients). Various reasons included cylinder values outside the range of the instrument (five patients), inability to keep the eyes focused on the target (two patients), uncontrolled blinking, head tremor, and confusion (one patient each). Further analysis of the eyes whose cylinder values could not be estimated by the AR revealed that on clinical refraction the cylinder values in dioptres ('plus') were $5 \cdot 0,5 \cdot 5,6 \cdot 25$, $6 \cdot 5$, and $7 \cdot 25$.

The percentage agreement between autorefraction and clinical refractive data with respect to the various refractive components is depicted in Table 1. More than $90 \%$ of the autorefraction values were within $0.51 \mathrm{D}$ of spherical equivalence, sphere, and cylinder powers. This figure rose to nearly $100 \%$ when only reliable readings on the AR were considered. $85 \%$ of the cylindrical axis values determined by the $\mathrm{AR}$ were within $11^{\circ}$ of clinical refraction, improving to $90 \%$ with reliable measurements alone. The percentage agreement between the AR and clinical refractive data in the reliable and less reliable autorefractive data groups was compared by the $\chi^{2}$ test. This revealed that there was a statistically significant difference between the above two AR groups in spherical equivalence $(p<0.05)$, sphere power $(\mathrm{p}<0.005)$, cylinder power $(\mathrm{p}<0.001)$, and cylinder axis $(\mathrm{p}<0.05)$.

In seven eyes a cylinder value was present in one of the two methods of refraction (that is, either on the AR or during clinical refraction) but not in the other. Aurorefraction indicated a cylinder value in six of these eyes (three in the reliable group and three in the less reliable group) where none was found on clinical testing. A single eye needed cylindrical correction during subjective verification, but this was not displayed by the AR.

The mean differences between autorefraction and clinical refraction in spherical equivalence, sphere power, cylinder component, and cylinder axis are shown in Table 2. Spherical equivalence and sphere component were both more myopic or less hypermetropic on the AR in comparison with conventional refraction, unlike the cylinder power, which was more hypermetropic and less myopic. The mean difference in cylinder axis was $4 \cdot 7^{\circ}\left(\mathrm{SD} 6 \cdot 1^{\circ}\right)$.

Autorefractive data were categorised into reliable and less reliable values. The differences between mean reliable $A R$ values and clinical refraction data were computed and compared with values derived in a similar fashion for less 
reliable data. Tested by the standard error of the difference between the means to assess statistical significance, the two groups showed no difference in spherical equivalence $(p>0.9)$, sphere power $(\mathrm{p}>0.7)$, or cylinder power $(\mathrm{p}>0.6)$. However, the mean difference in cylinder axis was significant $(p<0.0001)$.

\section{Discussion}

Extracapsular cataract extraction followed by posterior chamber IOL implantation is the current method of choice in the visual rehabilitation of cataract patients. With preoperative ocular biometry the surgeon can achieve the desired postoperative refraction in a significant number of patients. ${ }^{17}$ However, ocular refraction will continue to play an important part in the postoperative evaluation and visual rehabilitation of pseudophakic eyes until bifocal or multifocal IOLs achieve their promised potential.

An autorefractometer which can be operated by the nursing staff to provide accurate refraction values in pseudophakes will result in an appreciable saving of the clinician's time. Previous reports on autorefraction in pseudophakic eyes have not been adequately comprehensive. Rassow and Wesemann' classified results as good, bad, or instrument failure in terms of the visual acuity achieved with the autorefractor reading compared with subjective refraction. Wood ${ }^{10}$ found that IOLs accounted for a significant number of unobtainable or rejected AR measurements. Dufier $e t a l^{2}$ studied a general clinic population which included normal, aphakic, and pseudophakic eyes, but pseudophakes were not separately analysed.

In this study the various autorefractive data in pseudophakic eyes were all acceptably accurate, especially as preliminary refraction. Subjective verification of this result is essential before final prescription. A similar outcome with the AR has been experienced in normal patients ${ }^{14}$ and children $^{15}$ under cycloplegia. In comparison, manifest autorefraction in normal subjects has yielded poorer results for spherical equivalence, with percentage agreement with clinical refraction being as low as $41 \% .^{12}$ This is probably because the in-built autofogging mechanism in the AR fails to neutralise adequately the patient's accommodation, producing significant instrument myopia. ${ }^{14}$ This phenomenon does not affect aphakes or pseudophakes who lack accommodation, accounting for autorefraction results which are superior to those observed in subjects with intact accommodation..$^{24101214}$

Although differential analysis of the reliable and less reliable AR reading showed a statistically significant difference between the two groups, this was not clinically important,${ }^{14}$ as the autorefractive data were considered as preliminary results needing subjective verification.

It is not only the accuracy of the autorefraction measurements that is important but also the percentage of a given number of eyes that can be measured with an AR. AR readings were either invalid or not obtainable in $9 \%$ (10 out of 110 ) of the eyes eligible for study where conventional refraction was possible. This is lower than the $25 \%{ }^{10}$ and $60 \%{ }^{4}$ mentioned in previous studies on autorefraction which have included pseudophakic eyes.

Five eligible eyes $(5 \%)$ could not be measured by the AR because the cylinder values were supposedly outside its specified range. On subjective refraction all but one eye had a cylinder power of less than $+7 \mathrm{D}$. Possibly the patients with these eyes had difficulty in accepting subjectively the high powered cylindrical correction, and hence the cylindrical power determined clinically was less than that indicated objectively by autorefraction. It is also possible that the AR tended to overestimate the cylinder component, as this was skewed towards more plus or less minus in comparison with clinical refractive data. The routine clinical practice in our unit is to adjust or remove corneal sutures after eight weeks if there is a cylindrical correction greater than $2.5 \mathrm{D}$. The five eyes mentioned above had their corneal sutures removed, but subsequent $A R$ values have not been included in this study.

Six of the study eyes which had a cylinder component on the AR did not need it on subjective verification. Ghose et $a l{ }^{7}$ in their study of autorefraction in normal subjects, have pointed out that cylinder values obtained on the AR were often clinically insignificant.

The clinical impression gained during this evaluation was that autorefraction was both quick and easy to perform. Although this procedure was not timed and compared with conventional refraction, a saving in the clinician's time of four minutes per eye has been reported in a clinic setting.

In conclusion, it is suggested that an AR with a special facility for refracting pseudophakic eyes has a useful role to play in the routine evaluation of pseudophakes, particularly if these values are treated as preliminary refraction data requiring subjective verification.

We wish to thank Sister Anne Lewis for administrative help and Miss Lisa Jones for secretarial assistance.

1 Rassow B, Wesemann W. Automatic infra-red refractors. Ophthalmology 1984; 91(S): 10-26.

2 Dufier JL, Abitbol M, Pigamo F, Prete T, Paris JP Poitreuand $O$. Automatic objective refractometer: evalua tion in 3618 eyes. $F$ Fr Ophtalmol $1987 ; 10: 301-8$.

3 Guyton DL. Automated refractors - 1983. Ophthalmology 1983; 90(9S): $36-44$.

4 French CN, Wood ICJ. The Dioptron in practice. Optician 1981; 181: 18-30.

5 Wong EK, Patella V, Pratt M, Myers S, Gaster R, Irving HL. Clinical evaluation of the Humphrey automatic refractor. Arch Ophthalmol 1984; 102: 870-5.

6 Wood ICJ, Papas E, Burghardt D, Hardwick G. A clinica evaluation of the Nidek autorefractor. Ophthalmic Physio Opt 1984; 4: 169-78.

7 Ghose S, Nayak BK, Singh JP. Critical evaluation of the NR 1000F autorefractometer. Br F Ophthalmol 1986; 70: 221-6.

8 Reddy NS, Agarwal S. Clinical evaluation of the Topcon autorefractor. Indian $\mathcal{F}$ Ophthalmol 1987; 35: 407-10.

9 Wesemann W, Rassow B. Automatic infra-red refractors comparative study. Am $\mathcal{f}$ Optom Physiol Opt 1987; 64: 627comparative study. Am F Optom Physiol Opt 1987; 64: 62738

10 Wood ICJ. A review of autorefractors. Eye 1987; 1: 529-35. 11 Anonymous. Refractors. Ophthalmology 1988; 95(S): 171-6.

12 Ehrlich DL. The Canon RK-1: refractor mode evaluation Ophthalmic Physiol Opt (in press).

13 Guillon M. Automated refraction in aphakia-II. Its repeatability and accuracy compared to conventional techniques. Ophthalmic Physiol Opt 1986; 6: 85-9.

14 Nayak BK, Ghose S, Singh JP. A comparison of cycloplegic and manifest refractions on the NR-1000F (an objective autorefractometer). Br $\mathcal{F}$ Ophthalmol 1987; 71: 73-5.

15 Hunold W, Auffarth G, Effert R. Clinical application of the Canon R-10 autorefractor in children with squint. Klin Canon R-10 autorefractor in children

16 Charman WN. Infra-red autorefractors - here to stay? Ophthalmic Physiol Opt 1985; 5: 237-9.

17 Dang MS, Sunder Raj PP. SRK II formula in the calculation of intraocular lens power. Br f Ophthalmol 1989; 73: 823-6. 